\title{
WSRT HI synthesis absorption study towards Cassiopeia A
}

\author{
U.J. Schwarz ${ }^{1}$, W.M. Goss ${ }^{2}$, and P.M.W. Kalberla ${ }^{3}$ \\ 1 Kapteyn Astronomical Institute, Groningen University, Postbus 800, 9700 AV Groningen, The Netherlands \\ 2 National Radio Astronomy Observatory, P.O. Box O, Socorro, NM 87801, U.S.A. \\ 3 Radioastronomisches Institut, Universität Bonn, Auf dem Hügel 71, D 53121 Bonn 1, Germany
}

Received May 23; accepted August 28, 1996

\begin{abstract}
We present high resolution images of the entire galactic HI spectrum of Cas A and display the whole data cube in various ways; the spatial resolution is 30 arcsec and the velocity resolution is $0.62 \mathrm{~km} \mathrm{~s}^{-1}$. In previous studies, observations are described either with lower velocity resolution or with a limited velocity range (below $-30 \mathrm{~km} \mathrm{~s}^{-1}$ ), (Bieging et al. 1991; Reynoso et al. 1997). Here we concentrate the discussion on the local HI gas, with velocities between -10 and $+10 \mathrm{~km} \mathrm{~s}^{-1}$. At a velocity of $-1.5 \mathrm{~km} \mathrm{~s}^{-1}$ we find a cold $\mathrm{HI}$ feature with a kinetic temperature of typically $25 \mathrm{~K}$, with a small region of 1 by 2 arcmin with a kinetic temperature below $10 \mathrm{~K}$. This feature can be associated with a ${ }^{13} \mathrm{CO}$ feature observed by Wilson et al. (1993). Based on the new HI absorption results we derive the ratio $N_{\mathrm{HI}} / N\left(\mathrm{H}_{2}\right) \approx 0.05$.
\end{abstract}

Key words: ISM: Cas; clouds atoms — radio lines: ISM

\section{Introduction}

Using HI absorption observations in front of extended continuum sources it is possible to study the small scale structure of the cold galactic HI. Cas A with a total flux density of $2100 \mathrm{Jy}$ and a diameter of 5 arcmin provides high sensitivity because of the high surface brightness of the source. The source has been observed previously with various interferometers in HI. The present observations have an angular resolution of $30^{\prime \prime}$ and a velocity resolution of $0.6 \mathrm{~km} \mathrm{~s}^{-1}$. Previous observations of Cas A were observed with lower resolution and sensitivity using the Caltech interferometer (Greisen 1973) as well as the WSRT. The latter observations of Cas A were done to measure the HI Zeeman splitting (Schwarz et al. 1986). More recently Bieging et al. (1991) observed the HI absorption against Cas A with the VLA with high angular and velocity resolution $\left(7^{\prime \prime}, 0.6 \mathrm{~km} \mathrm{~s}^{-1}\right)$; however the velocity range was limited to the "Perseus feature" $\left(-55<v<-30 \mathrm{~km} \mathrm{~s}^{-1}\right)$;

Send offprint requests to: U.J. Schwarz the local velocity, $v>-30 \mathrm{~km} \mathrm{~s}^{-1}$ ("Orion" arm) was not observed. Their results show a wealth of fine structure. The purpose of the present publication is to present high resolution images of the entire galactic HI spectrum of Cas A. We concentrate however our discussion on the local velocities $\left(>-30 \mathrm{~km} \mathrm{~s}^{-1}\right)$.

Our results can be used also to compare with obervations of molecular lines (e.g. Wilson et al. 1993), recombination lines (Anantharamaiah et al. 1994), but also with optical, IR and X-ray (Keohane et al. 1996) images of Cas A. The latter authors have used the WSRT data to calibrate the soft X-ray absorption in the direction of Cas A.

Small scale structure with resolutions similar to the present one were also found toward other sources, such as 3C 58 (Roberts et al. 1994), W3 (van der Werf \& Goss 1989), 3C 10 (Schwarz et al. 1995), Orion (Lockart \& Goss 1978; van der Werf \& Goss 1990), W49 (Lockart \& Goss 1978).

Cas $\mathrm{A}$ is at galactic longitude $112^{\circ}$ and at latitude $-2^{\circ}$. The line of sight samples the galaxy without velocity ambiguity in the second quadrant; Cas A is believed to lie on the far side of the Perseus arm, which has a distance of $\sim 3 \mathrm{kpc}$ from the sun (Read et al. 1995). At low velocities the HI has typically a distance of $100 \mathrm{pc}$ ( the thickness of the cold HI layer); the angular resolution corresponds then to $0.014 \mathrm{pc}$. At velocities of the Perseus arm $(-52<v<$ $-34 \mathrm{~km} \mathrm{~s}^{-1}$ ) with a distance of $3 \mathrm{kpc}$ the linear resolution is $0.5 \mathrm{pc}$.

The spin temperature could in principle be deduced from the comparison of absorption features seen at the edge of the source with HI emission just outside the source. But no attempts were made to image the emission outside the source because of the confusion by the 10 arcmin grating response and the low sensitivity of $18 \mathrm{~K}$ at the original resolution. Moreover the strong sidelobes of this extended source with varying absorption as a function of velocity introduces additional uncertainties. Although the direct determination of the spin temperature is excluded, we can provide upper limits from the velocity widths of 
the features. Knowing the angular size of the HI concentrations and their distance, estimates of the space density can be made for a given spin temperature.

The main part of the paper consists of the display of data-cube of optical depths at all velocities. First, we give series of "channel-images" (= maps at constant velocity), then we show cuts through the cube at constant declinations and finally a series of individual spectra. In the last section we give a short discussion of some of the results.

\section{Observations}

The observations were obtained from the Westerbork Synthesis Telescope (WSRT), using the 5120 channel digital backend. The main observational parameters are summarized in Table 1.

Table 1. Observational parameters of WSRT HI observations of Cas A

Observing dates:

Total Observing time:

Spacings:

Grating ring:

Primary beam HPBW:

Phase and pointing

centres:

Synthesized beam HPBW:

Grid spacing:

Spectrometer:

rms noise:

Line

$\tau$ (if $\tau \ll 1$ ) mean

Continuum

Conversion

Continuum brightness:

Maximum

Minimum within shell

Average ( $>2.5 \mathrm{Jy} /$ beam $)$

August, November 1979

$2 \times 12 \mathrm{hrs}$

$36 \mathrm{~m}(171 \lambda), 108 \mathrm{~m}(515 \lambda) \ldots \tau(v)=-\ln \left(1+\frac{T_{v}-T_{\mathrm{c}}}{T_{\mathrm{c}}+T_{\mathrm{g}}-T_{\mathrm{s}}}\right)$
to $1260 \mathrm{~m}(5972 \lambda)$

in increments of $72 \mathrm{~m}(343 \lambda)$

Grating ellipse with a radius

of $11^{\prime}$ in declination

and $10^{\prime}$ in right ascension

$36^{\prime}$

$\alpha(1950)=23^{\mathrm{h}} 21^{\mathrm{m}} 07.0^{\mathrm{s}}$;

$\delta(1950)=58^{\mathrm{h}} 32^{\prime} 47^{\prime \prime}$

$(\ell=111.73, b=-2.12)$

$(\alpha \times \delta)=27^{\prime \prime} \times 32^{\prime \prime}$

$9.84^{\prime \prime} \times 9.84^{\prime \prime}$

255 velocity channels.

Total velocity width

$132 \mathrm{~km} \mathrm{~s}^{-1}(0.625 \mathrm{MHz})$

$V_{\text {lsr }}=-105 \mathrm{~km} \mathrm{~s}^{-1}$ to

$23 \mathrm{~km} \mathrm{~s}^{-1}$

Channel separation

$0.52 \mathrm{~km} \mathrm{~s}^{-1}$

Velocity resolution

$0.62 \mathrm{~km} \mathrm{~s}^{-1}$

$70 \mathrm{mJy} /$ beam $(18 \mathrm{~K})$

0.014

$7.5 \mathrm{mJy}(1.8 \mathrm{~K})$

$1 \mathrm{mJy} /$ beam $=0.25 \mathrm{~K}$

$20.6 \mathrm{Jy} /$ beam $(5000 \mathrm{~K})$

$4.2 \mathrm{Jy} /$ beam $(1000 \mathrm{~K})$

$5 \mathrm{Jy} /$ beam $(1250 \mathrm{~K})$

The noise in the observations is higher by a factor two than expected, since Cas A $\left(T_{\mathrm{a}}=225 \mathrm{~K}\right.$, aperture beam efficiency $=0.64)$ as well the HI background emission contribute to the system temperature (in $1979 T_{\text {sys }}=90 \mathrm{~K}$ ). Because the $\mathrm{HI}$ absorbs the continuum radiation in some channels partly or even almost completely, the above effect is reduced and the noise in these maps is lower, i.e. the noise varies from channel to channel. In the analysis we did not take these variations into account.

The data were calibrated using the standard procedures provided by WSRT staff in Dwingeloo. The maps were not cleaned. Experiments showed that the effects of the sidelobes of the synthesized beam were not large (below the noise), with the exception of channels with are partly strongly absorbed. But due to the "corrugation"effect (Schwarz 1984), additional uncertainities are introduced by cleaning, and some systematic effects for large optical depth have to be taken into account, typically $15 \%$. The Groningen GIPSY software package was used to analyze the data and to prepare all figures.

The continuum radiation of the source is derived by averaging channels which are free of line emission; the continuum image is displayed in the first panel of Fig. 2. Optical depths were computed according Eq. (1).

where $T_{\mathrm{s}}$ is the spin temperature of $\mathrm{HI}$ and $T_{v}, T_{\mathrm{c}}, T_{\mathrm{g}}$ and the galactic background (including HI-emission), respectively. The continuum intensity has a peak value of $20.6 \mathrm{Jy} /$ beam. Opacities were calculated only for regions of the source with a brightness above a cut-off of $T_{\mathrm{c}}$ of $1.25 \mathrm{Jy} /$ beam. The effects of $T_{\mathrm{s}}$ and $T_{\mathrm{g}}$ are described in Schwarz et al. 1995; since these quantities are not known and are estimated to be much smaller compared than $T_{\mathrm{c}}$ (see Table 1), we neglect these terms. The errors in $\tau_{v}$ depend on the rms noise, $\sigma$, of the observations and on $T_{\mathrm{c}}$. For small optical depths, the errors are $\sigma / T_{\mathrm{c}}$. This error is displayed in Fig. 2, in the second panel; in Table 1 we give the value for the average continuum $\bar{T}_{\mathrm{c}}$. For large optical depths the errors are determined by noise and by the spectral dynamic range (channel to channel calibration errors) of 1000 . The maximum optical depth reliably measured is $\approx 5$.

\section{Presentation of the data}

First we show in Fig. 1 the spectrum of the average optical depth across the source; this corresponds roughly to single-dish observations. Low level features are enhanced by giving a scaled-up version of the profile (thin line).

The display of a large data-cube without colour is a well-known difficulty. Three main displays are utilized, with all intensities as optical depths:

(i) Fig. 2 shows "channel images", i.e. images in right ascension and declination for adjacent velocities. Optical are the brightness of the line radiation, the continuum 


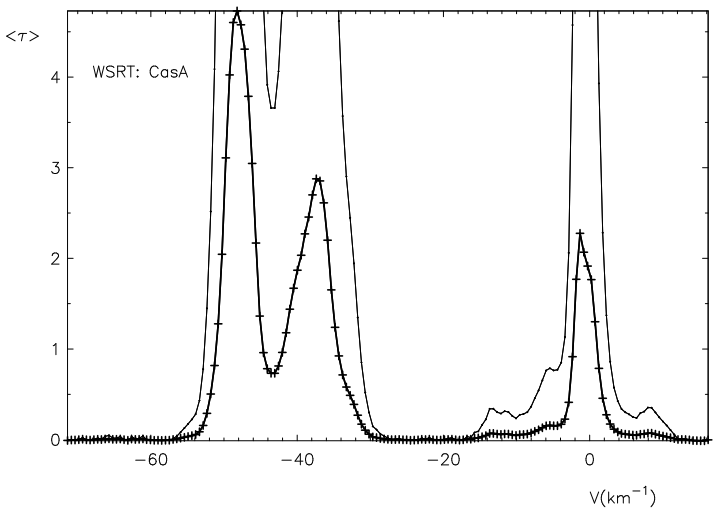

Fig. 1. Average optical depth spectrum of Cas A. The thin line is the spectrum scaled by a factor 5

depths below the (one sigma) error limit $\sigma / T_{\mathrm{c}}$ were omitted; these uncertainties are displayed as an error map in the second panel. The third panel is a map of the "equivalent width" in HI, $\int \tau \mathrm{d} v$, which can be used for correlations with X-ray data, etc; the units are $\mathrm{km} \mathrm{s}^{-1}$.

(ii) In Figs. 3 and 4 we show velocity-right ascension images (horizontally the velocity, vertically the right ascension) for a series of declinations, for two velocity ranges.

(iii) Figs. 5 and 6: Series of spectra arranged in the same order as in the velocity-right ascension maps.

All figures are based on coordinates of right ascension and declination. Galactic coordinates are included in Fig. 9.

\section{Discussion}

\subsection{Perseus arm feature $\left(-55<v<-30 \mathrm{~km} \mathrm{~s}^{-1}\right)$}

As mentioned in the introduction, the VLA observations presented by Bieging et al. (1991), have a similar velocity resolution, but have a factor $\approx 4$ higher angular resolution as compared with that of the present data. There is good qualitative agreement. The distinction of four velocity intervals by Bieging et al. (1991), with characteristic morphology of the HI absorption ("curtain", "floccules", "arc and loops" and "linear filaments") are not as obvious in our observations; this effect arises due to the inferior angular resolution here.

The velocity-right ascension diagram (Fig. 3) is adequate to show the large scale velocity behaviour of the absorbing cool HI, such as gradients and "bends" (as at $\Delta \delta=+79^{\prime \prime}, v=-35 \mathrm{~km} \mathrm{~s}^{-1}$ ). Due to the undersampling of the contours, details are less visible. For these Figs. 5 with the collection of spectra are more suitable, e.g. there is a strong feature seen at $\Delta \delta=-20^{\prime \prime}, \Delta \alpha=+160^{\prime \prime}\left(5^{\mathrm{s}}\right)$, $v=-43 \mathrm{~km} \mathrm{~s}^{-1}$.

The physical interpretation of the saturated line $(\tau \approx 5)$ at $v=-50 \mathrm{~km} \mathrm{~s}^{-1}$ is complex; in spite of the saturation, we can derive some limits. The absorption line could be caused by a large scale feature of high density and a spin temperature, $T_{\mathrm{s}}=50 \mathrm{~K}$; this latter value was estimated as follows: first we fitted Gaussians to the optical depth profiles, thereby disregarding the residuals of these velocities, where the line is saturated. We derive a peak optical depth of about 6 , with a velocity width (FWHM) of $3.8 \pm 0.6 \mathrm{~km} \mathrm{~s}^{-1}$. From this width we find an upper limit for the kinetic temperature of $310 \mathrm{~K}$ using

$$
T_{\mathrm{k}}=21.8 \Delta v_{1 / 2}^{2} .
$$

The spin temperature $T_{\mathrm{s}}$ is probably considerably lower because single dish observations give a brightness temperature of about $50 \mathrm{~K}$ (Mebold \& Hills 1975); therefore $T_{\mathrm{s}}$ is about the same for a cloud of such high optical depth. The integral of the optical depth over the velocity is $25 \mathrm{~km} \mathrm{~s}^{-1}$, which results in a value of the column density $N_{\mathrm{HI}}=4510^{18} T_{\mathrm{s}} \mathrm{cm}^{-3}$. If we assume that the feature has a diameter of 5 arcmin (the moving "curtain" across the source) and that it is the same in the line of sight, the space density is $8 T_{\mathrm{s}} \approx 400 \mathrm{~cm}^{-3}$. Another interpretation would be that the saturated line is the blend of many small scale features of very cold HI. The rather regular morphology of the feature at $-50 \mathrm{~km} \mathrm{~s}^{-1}$ makes the latter explanation less attractive.

\subsection{The local gas $\left(-10<v<10 \mathrm{~km} \mathrm{~s}^{-1}\right)$}

The velocity-right ascension diagram (Fig. 4) gives the impression of uniformity; even the spectra (Fig. 6) seem to confirm this impression. However in the channel images several concentrations are visible; one of these is at $\alpha=23^{\mathrm{h}} 25^{\mathrm{m}}, \delta=58^{\mathrm{o}} 33^{\prime}$. In order to study the small scale structure, we computed images by subtracting the average optical depth from the channel images. In Fig. 7 we display the spectra based on these images. In these spectra the feature mentioned above is clearly visible.

Gaussian fitting of this narrow feature gives the following average parameters: integral $\tau=0.90 \mathrm{~km} \mathrm{~s}^{-1}$, $v=-1.31 \mathrm{~km} \mathrm{~s}^{-1}$, observed velocity width (FWHM) $\Delta v_{1 / 2}=0.84 \pm 0.15 \mathrm{~km} \mathrm{~s}^{-1}$. The velocity width (FWHM) corrected for instrumental broadening of $0.62 \mathrm{~km} \mathrm{~s}^{-1}$ gives an average of $\Delta v_{1 / 2}=0.49 \pm 0.38 \mathrm{~km} \mathrm{~s}^{-1}$. The small deconvolved value of the velocity width corresponds to an very low kinetic temperature $T_{\mathrm{k}}=5.3 \mathrm{~K}\left(>T_{\mathrm{s}}\right)$ using Eq. (2). The large scatter in the corrected velocity width is due to the fact, that the correction is more uncertain in cases where the fitted velocity widths are only slightly larger than the instrumental resolution. For such a narrow feature the velocity resolution is barely adequate. The scatter is strongly influenced by the most narrow lines, whereas the average width is more reliably determined as well as the kinetic temperature. However the question arises, whether this narrow velocity feature is an isolated entity in space or if it is imbedded in a larger structure. 
In order to investigate this latter possibility we have attempted to isolate the narrow velocity feature from the broader feature at $\approx 0 \mathrm{~km} \mathrm{~s}^{-1}$ in the original spectra by Gaussian fitting. This fitting gave quite consistent results with the values derived above, mainly in velocity and velocity width. However characteristic of the fitting procedure of spectra of this type is that the broad component is affected by variations of the narrow component. This effect occured here which resulted in amplitudes with increased scatter. We tried a different approach to avoid this problem. First we fitted Gaussians to the average profile; three components gave a good fit (see Fig. 8): a narrow component at $-1.5 \mathrm{~km} \mathrm{~s}^{-1}$, the broader component at $0 \mathrm{~km} \mathrm{~s}^{-1}$ and the third, a very broad component of velocity width $12 \mathrm{~km} \mathrm{~s}^{-1}$. The latter two components represents portions of the spectra showing less variation across the source; then we subtracted these (mean) components from the original spectra. In the remaining spectra we fitted one component, the $-1.5 \mathrm{~km} \mathrm{~s}^{-1}$ feature. The images of the parameters of this component are shown in Fig. 9. In the upper two panels we show the the integral $\left(\int \tau\right)$ and the central velocity of the component. The $-1.5 \mathrm{~km} \mathrm{~s}^{-1}$ feature is present across the the whole source, with a strong maximum at the position of the narrow feature discussed above. The velocity is quite regular with a slight gradient. Only in the SE corner there is a region with different velocities. The halfwidth was corrected for instrumental broadening and then converted to the kinetic temperature $T_{\mathrm{k}}$, using Eq. (2). A clear minimum is visible with kinetic temperatures below $10 \mathrm{~K}$ at the position of maximum $\int \tau$. When we make the reasonable assumption that the spin temperature of the HI can be estimated to be close to $T_{\mathrm{k}}$, then $N_{\mathrm{HI}}$ can be computed by $1.83510^{18} \int \tau T_{\mathrm{s}}$. $N_{\mathrm{HI}}$ is shown in the last panel of Fig. 9. The strong peak in the image of $\int \tau$ disappears. The remaining fluctuations across the source are most unlikely to be significant. This analysis indicates that the narrow velocity feature is in fact only a cold $\left(T_{\mathrm{S}}<10 \mathrm{~K}\right)$ part of a larger, warmer $(\approx 25 \mathrm{~K})$ HI structure. This may well be the first detection of an HI feature with such a low spin temperature.

The depression in spin temperature could either be explained by the lack of heating (most likely by dust shielding the radiation field) or by a larger cooling rate (by additional heavy elements, which cause the cooling). Similar temperatures are found in molecular clouds, which are thought to be dense clouds and are shielded from the background uv-radiation (cf. Burton et al. 1978). Comparison with results of $\mathrm{CO}$ emission with a spatial resolution of $\approx 1^{\prime}$ (Troland et al. 1985; Wilson et al. 1993) with our synthesis HI absorption observations show no corresponding molecular cloud at the position of the low temperature HI. However there is a low velocity feature which seems to consist of both a narrow and broad component; the narrow component has a velocity of $-1.5 \mathrm{~km} \mathrm{~s}^{-1}$, exactly in agreement with the velocity of the HI cloud. In the observations of ${ }^{13} \mathrm{CO}$ by Wilson et al. (1993) there is an isolated narrow component at $-1.5 \mathrm{~km} \mathrm{~s}^{-1}$; this spectrum is included in Fig. 8 as dashed line; the agreement with the $\mathrm{HI}$ is striking. However there is limited spatial information available for a certain identification of the $\mathrm{CO}$ with the $\mathrm{HI}$ feature. The $\mathrm{H}_{2}$ column density derived by Wilson et al. (1993) is $410^{20} \mathrm{~cm}^{-2}$ and should now be compared with our average value of $N_{\mathrm{HI}}=0.210^{20} \mathrm{~cm}^{-2}$ for the $-1.5 \mathrm{~km} \mathrm{~s}^{-1}$ feature, instead of the single dish HI observations quoted in their paper. The ratio $N_{\mathrm{HI}} / N\left(\mathrm{H}_{2}\right)$ is then 0.05 and not two. The new value agrees roughly with the ratio observed for the clouds at velocities of the Perseus arm (0.1). A molecular line at $-1.5 \mathrm{~km} \mathrm{~s}^{-1}$ was also detected in CH by Rydbeck et al. (1976).

If the angular size $(\sim 2$ arcmin) of the narrow $\mathrm{HI}$ feature is also characteristic for the line of sight and we assume a reasonable distance of $100 \mathrm{pc}$, the thickness of the feature is $0.07 \mathrm{pc}$. From an average column density of $2010^{18}$ at $\mathrm{cm}^{-2}$, a space density of 100 at $\mathrm{cm}^{-3}$ follows. The total mass of the gas (including $\mathrm{H}_{2}$ ) of the structure is then $\approx 0.01 M_{\odot}$

Other small scale features are visible in Fig. 2. But due to the optimum scaling between minimum and maximum optical depth, these features are enhanced and are of much lower significance than the feature discussed above.

\subsection{The interarm region $\left(-26<v<-16 \mathrm{~km} \mathrm{~s}^{-1}\right)$}

At the velocities of the interarm gas $(-26<v<$ $-16 \mathrm{~km} \mathrm{~s}^{-1}$ ) there is almost no detectable absorption. However there is a possible small scale feature at $v=-19.9 \mathrm{~km} \mathrm{~s}^{-1}$ in the NE of the source.

\subsection{Extreme negative velocities $\left(v<-56 \mathrm{~km} \mathrm{~s}^{-1}\right)$}

Reynoso et al. (1997), have used the VLA to investigate HI absorption at velocities lower than the Perseus arm features. They find several "knots" with velocities $<-68 \mathrm{~km} \mathrm{~s}^{-1}$. We plotted these concentrations on top of our channel images in Fig. 10. There is in general good agreement, but not in detail; this is however not so surprising in view of the differencies in resolution (spatially higher and in velocity lower resolution than ours). These high velocity knots are interpreted as recombined wind driven clumps moving ahead of the SN shock front.

\section{Conclusions}

The main purpose of this paper is to give an overview of the complex structure of HI absorption towards Cas A. The data cube is displayed in various cuts. The "Perseus Arm" HI absorption was discussed in Bieging et al. (1991) based on their higher resolution VLA data. Our short discussion is therefore focussed on the local gas, previously not given in such detail. We found a 2 arcmin feature at a velocity of $-1.5 \mathrm{~km} \mathrm{~s}^{-1}$ of very small velocity width. 
This width corresponds to kinetic temperatures well below $10 \mathrm{~K}$. The mean profile of this feature corresponds with lines of molecules (as $\mathrm{CO}$ ) with the same velocity and velocity width. A new value of the ratio $N_{\mathrm{HI}} / N\left(\mathrm{H}_{2}\right)$ of 0.05 is derived.

Acknowledgements. We thank the staff of the WSRT for making the observations and the calibration of the data.The Westerbork Radio Synthesis Telescope is operated by the Netherlands Foundation for Research in Astronomy which is financially supported by the Netherlands Foundation for Scientific Research (NWO). The National Radio Astronomy Observatory is a facility of the National Science Foundation operated under cooperative agreement by Associated Universities, Inc.

We thank E.M. Arnal for help and the "GIPSY-team" in Groningen (K.G. Begeman, J.P. Terlouw and Martin Vogelaar) for their support and for providing many options in the plotting routines and J. Keohane (Minnesota), T. Troland (Kentucky), L. Rudnick (Minnesota) for the critical reading of the paper.

\section{References}

Anantharamaiah K.R., Erickson W.C., Payne H.E., Kantharia N.G., 1994, ApJ 430, 682

Bieging J.H., Goss W.M., Wilcots E.M., 1991, ApJS 75, 999 (BGW)
Burton W.B., Liszt H.S., Baker P.L., 1978, ApJ 219, L67

Greisen E.W., 1973, ApJ 184, 363

Kalberla P.M.W., Mebold U., Reich W., 1980, A\&A 82, 275

Keohane J.W., Rudnick L., Anderson M.C., 1996, ApJ 466, 309

Lockart I.A., Goss W.M., 1978, A\&A 67, 355

Mebold U., Hills D.L1., 1975, A\&A 42, 187

Read J.E., Hester J.J., Fabian A.C., Winkler P.F., 1995, ApJ 440, 706

Reynoso E.M., Goss W.M., Dubner G.M., Winkler P.F., Schwarz U.J., 1997, A\&A 317, 203

Roberts D.A., Goss W.M., Kalberla P.M.W., Herbstmeier U., Schwarz U.J., 1993, A\&A 274, 427

Rydbeck O.E.H., Kollberg E., Hjalmarson A., Sume A., Elldèr J., 1976, ApJS 31, 333

Schwarz U.J., 1984, in: "Indirect Imaging", 1984, Roberts J.A. (ed.). Cambridge Press, p. 255

Schwarz U.J., Troland T.H., Albinson J.S., et al., ApJ 301, 1986,320

Schwarz U.J., Goss W.M., Kalberla P.M., Benaglia P., 1995, A\&A 299, 193

Troland T.H., Crutcher R.M., Heiles C., 1985, ApJ 298, 808

v.d. Werf P.P., Goss W.M., 1989, A\&A 224, 209

v.d. Werf P.P., Goss W.M., 1990, A\&A 238, 296

Wilson T.L., Mauersberger R., Muders D., Przewodnik A., Olano C.A., 1993, A\&A 280, 221 


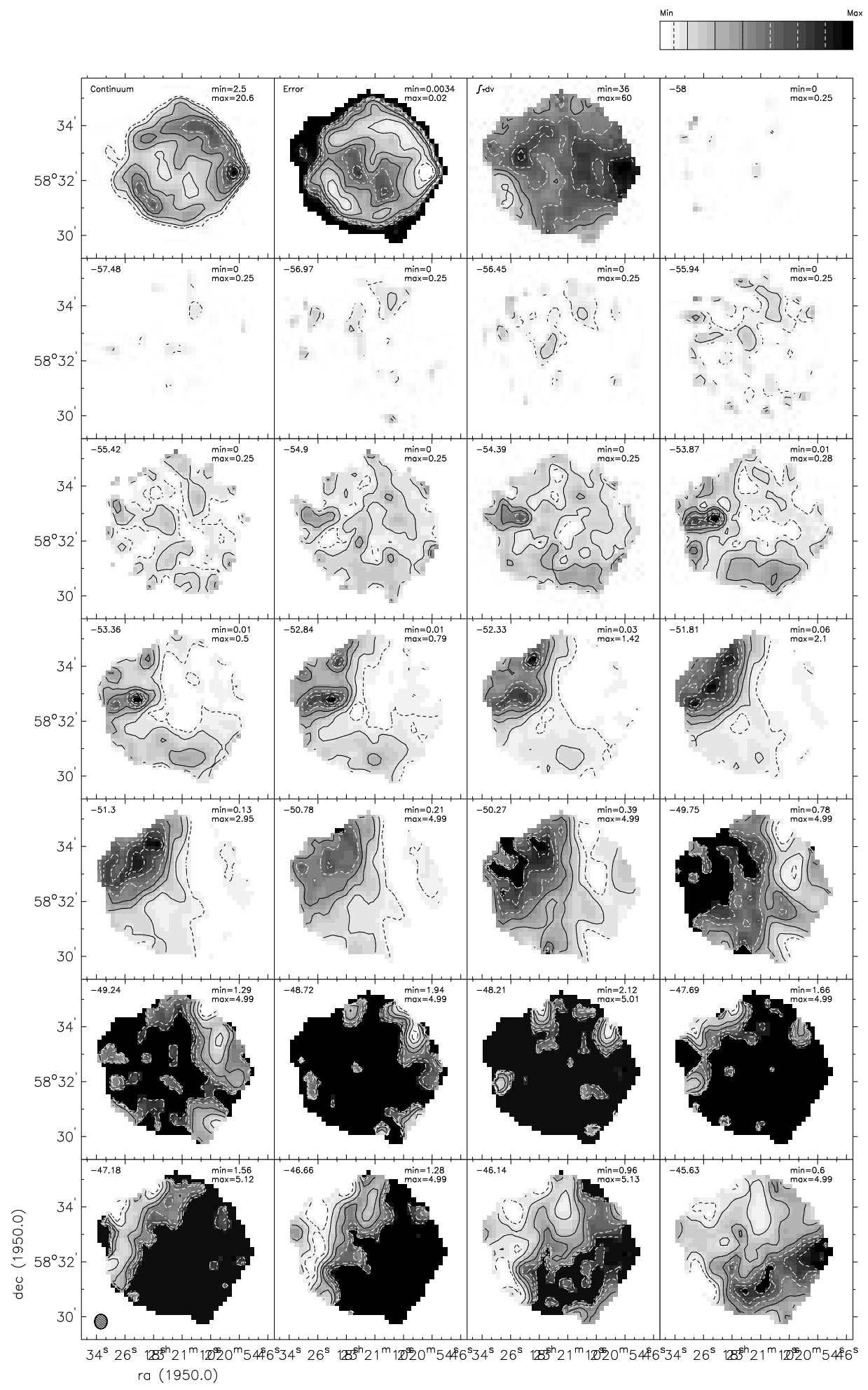

Fig. 2. HI Optical depth images for the velocities indicated. In the first frame the continuum image is shown (units Jy/beam), in the second frame the errors in $\tau$. The third panel is the map of the "equivalent width" in HI, $\int \tau \mathrm{d} v$; the units are $\mathrm{km} \mathrm{s}^{-1}$. The contours and grayscale are between the given minimum and maximum value as indicated in the individual plots 


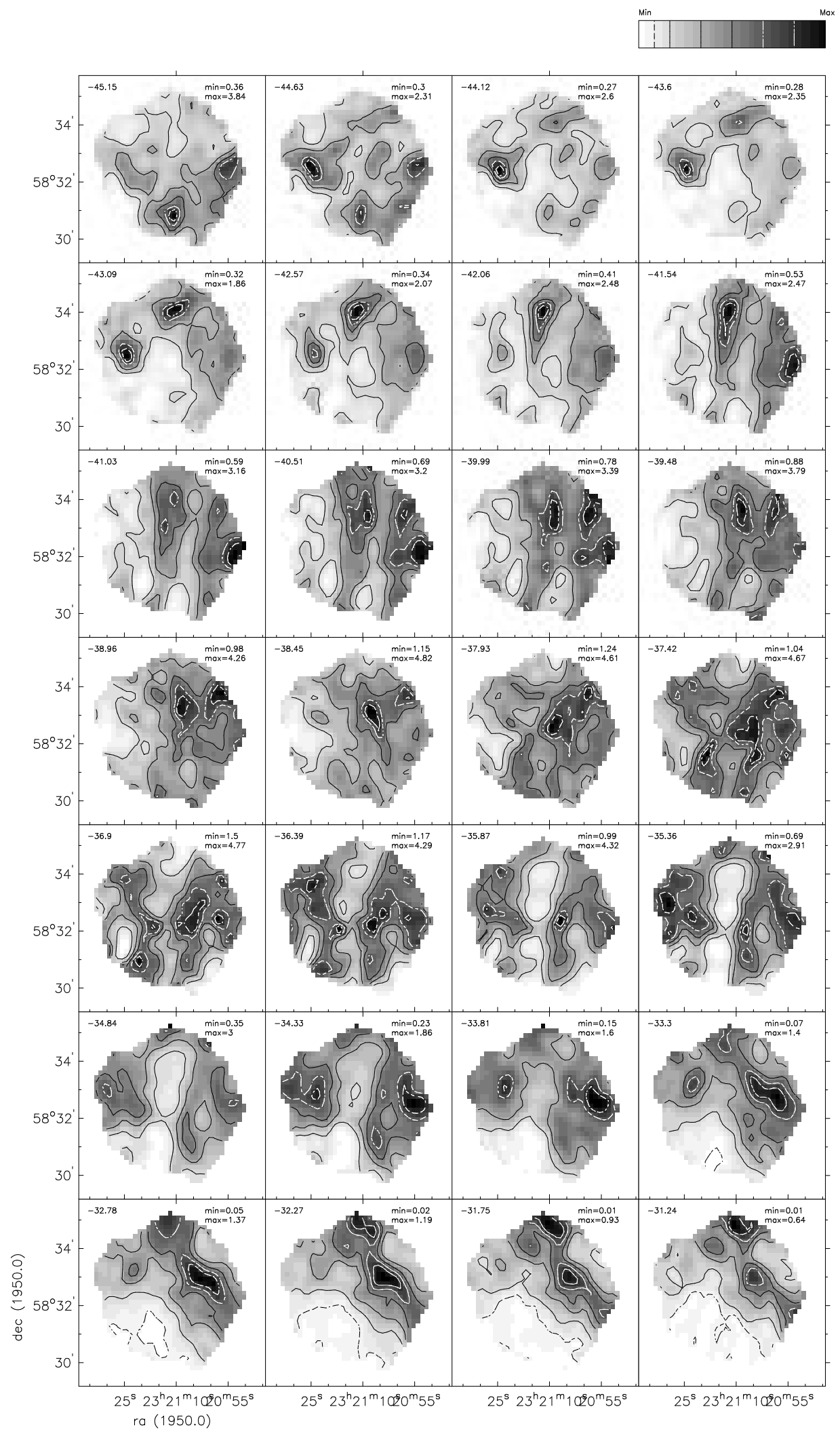

Fig. 2. a) 


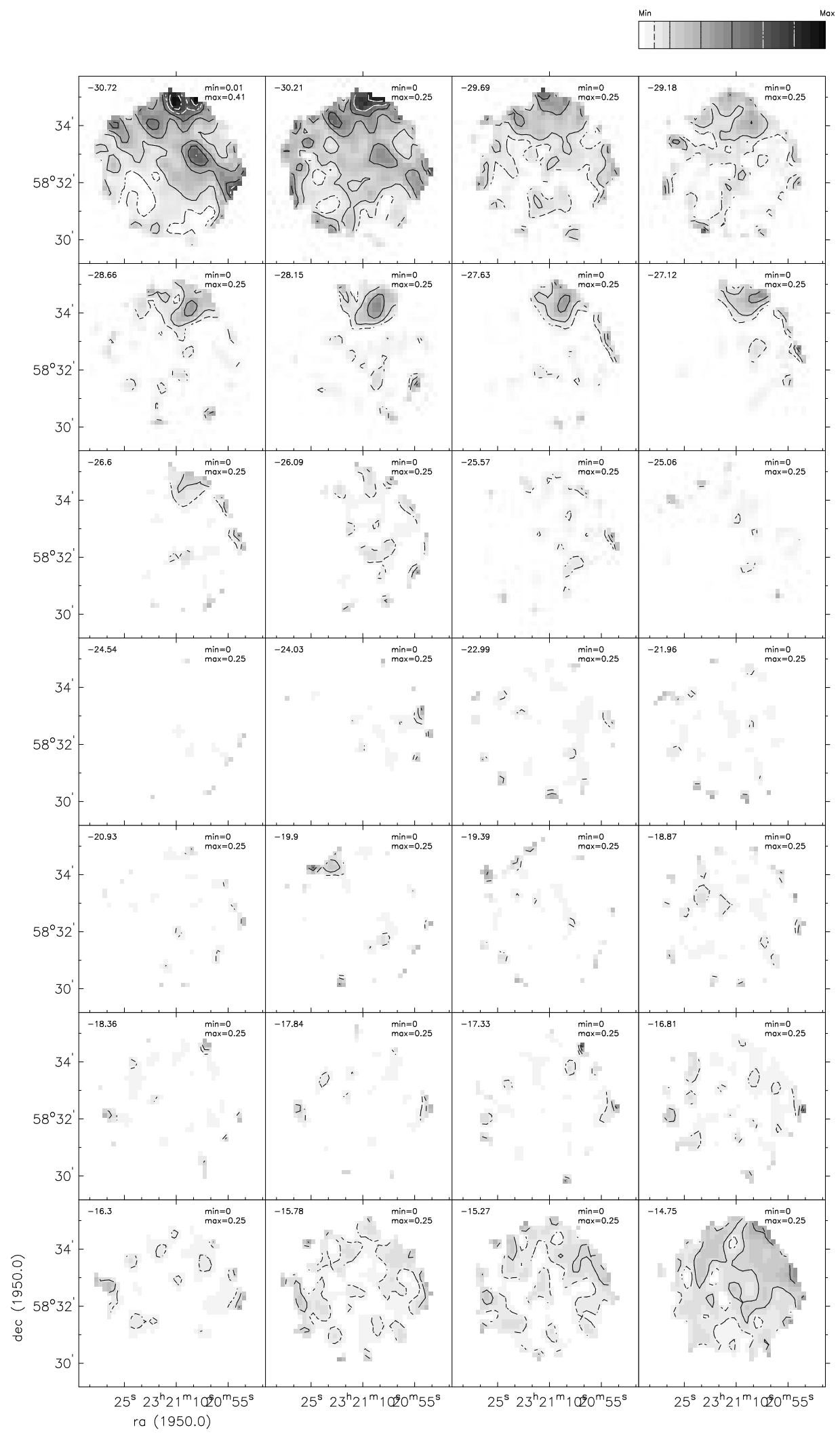

Fig. 2. b) 


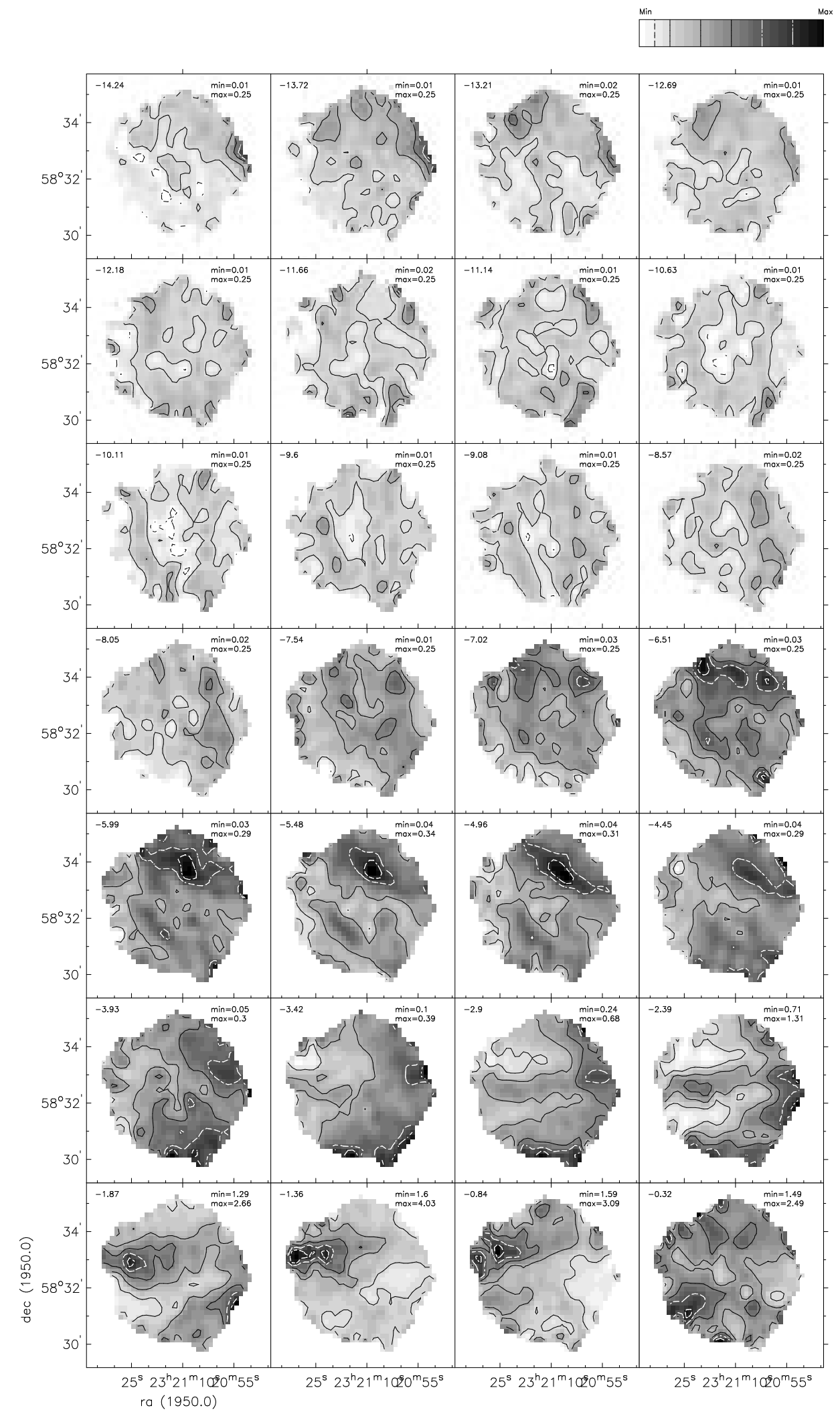

Fig. 2. c) 


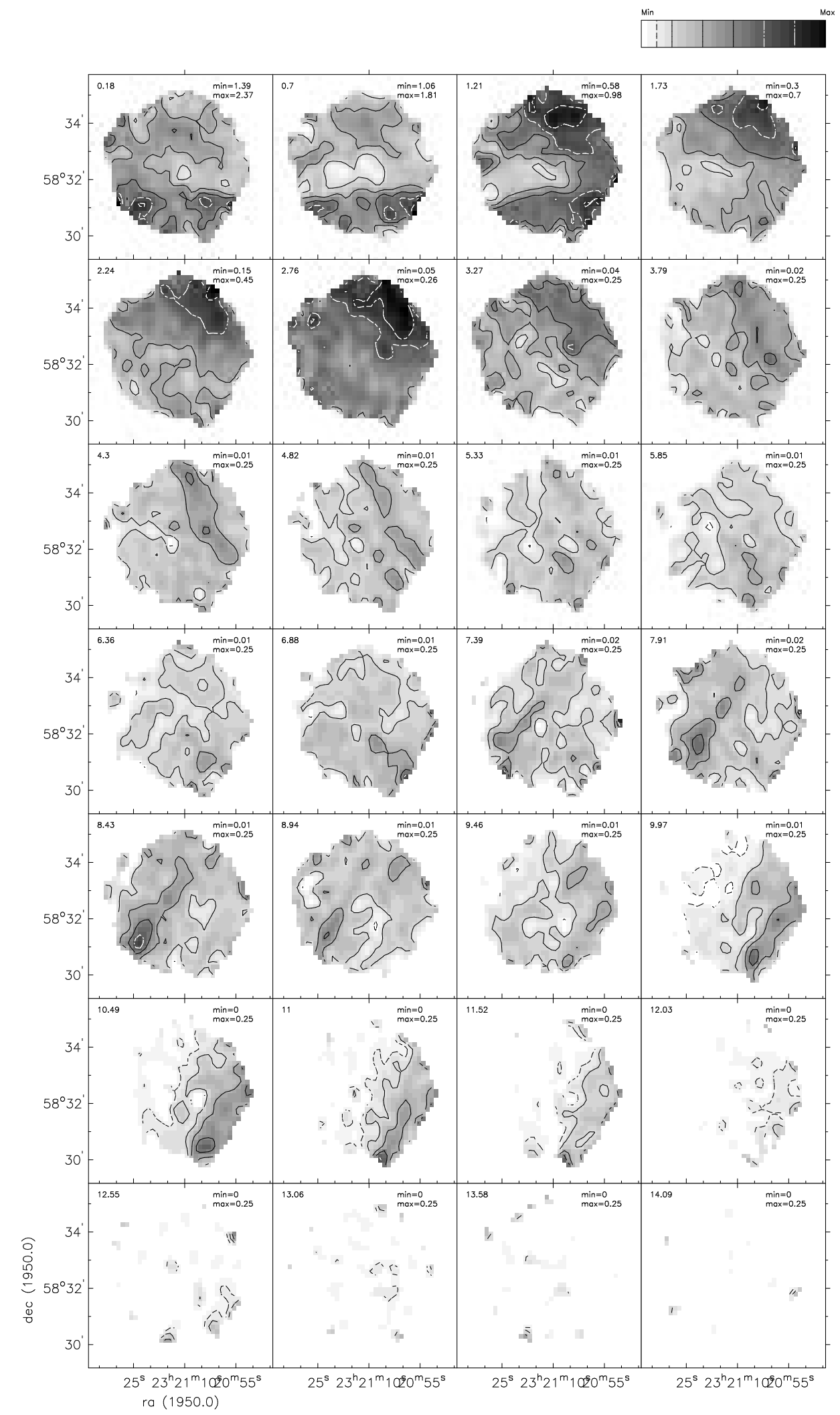

Fig. 2. d) 


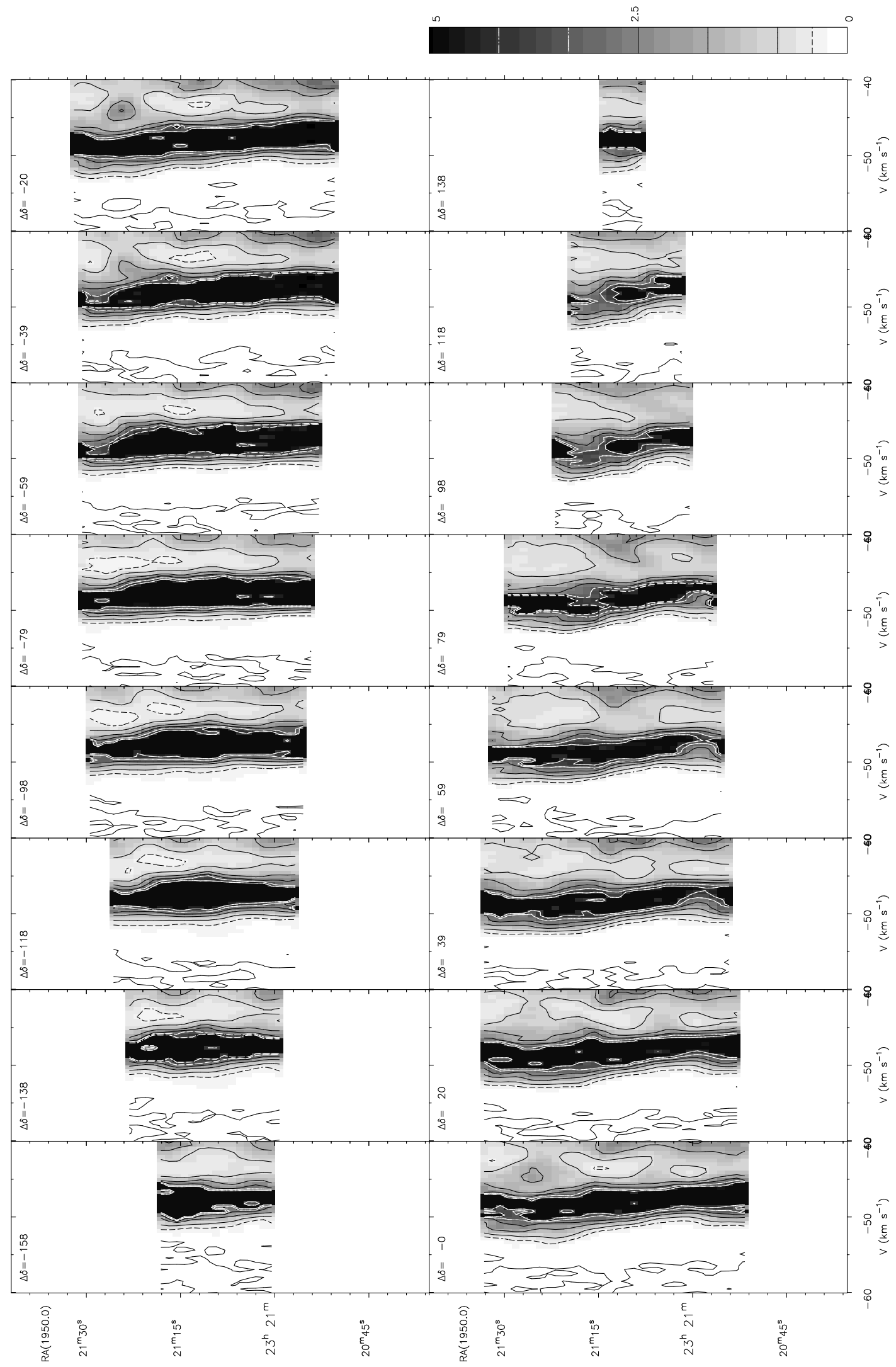

Fig. 3. Velocity-right ascension diagram of the Perseus arm HI feature optical depth in the interval -56 to $-30 \mathrm{~km} \mathrm{~s}^{-1}$, at various declinations given in offsets from phase and pointing centre (cf. Table 1) 


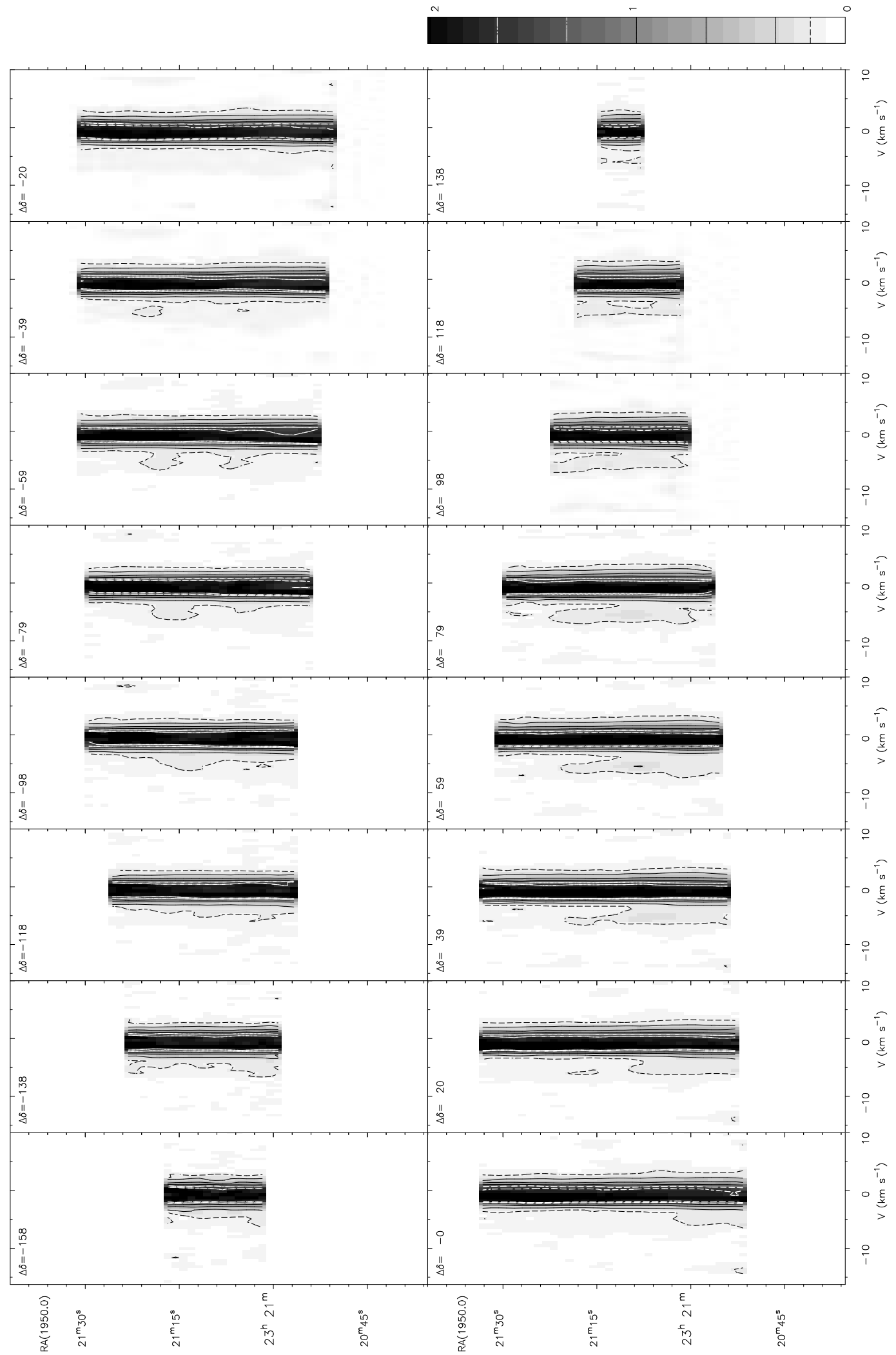

Fig. 4. Velocity-right ascension diagram of optical depth of the local gas, with velocities in the interval -16 to $10 \mathrm{~km} \mathrm{~s}^{-1}$, at various declinations given in offsets from phase and pointing centre (cf. Table 1) 


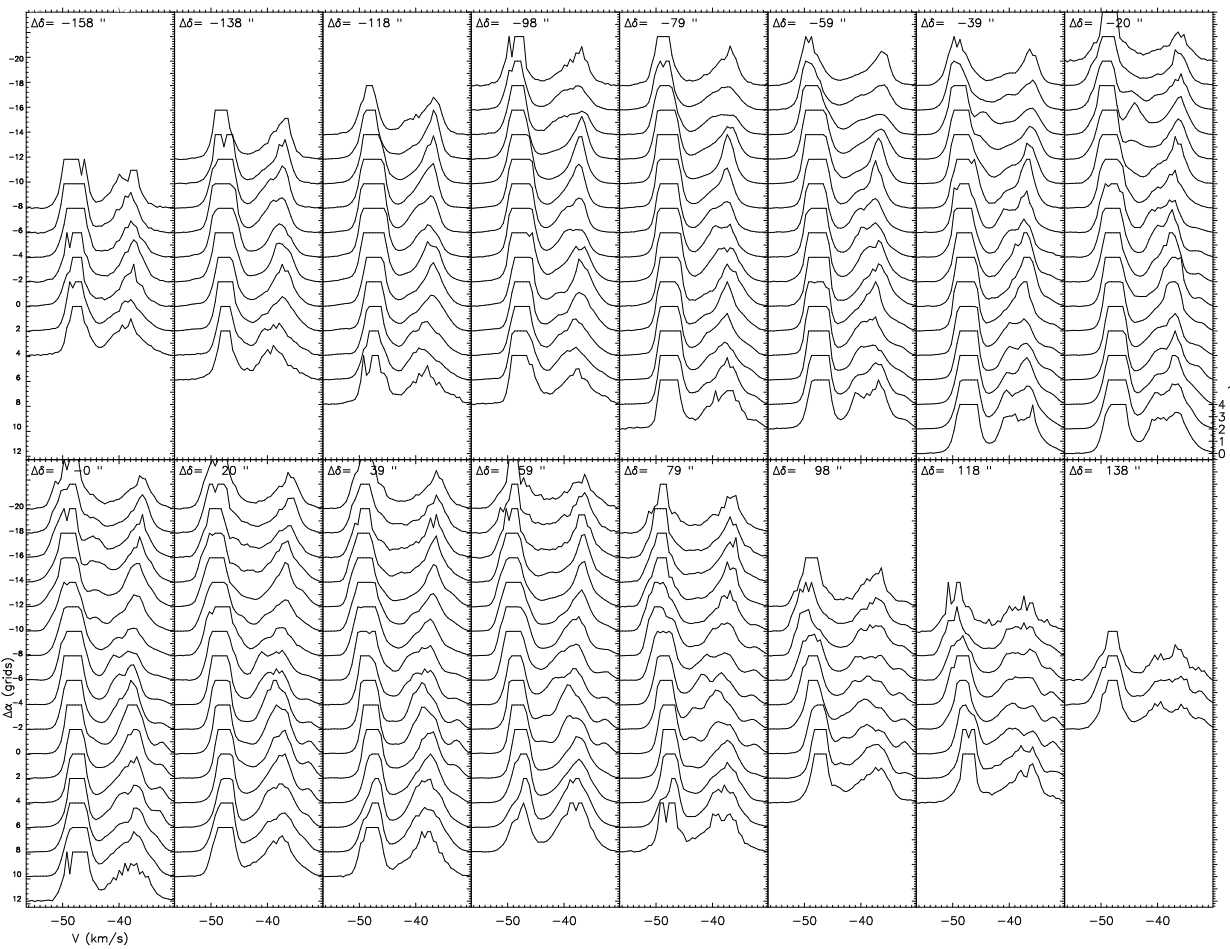

Fig. 5. HI spectra of the Perseus arm feature optical depth, in the interval -56 to $-30 \mathrm{~km} \mathrm{~s}^{-1}$. Only every second pixel of the grid is displayed in steps of $-19.7^{\prime \prime}$ (about $2 / 3$ beamsize). Offsets in right ascension and declination are with respect to the phase and pointing centre (cf. Table 1). The intensity scale is given in the top right panel

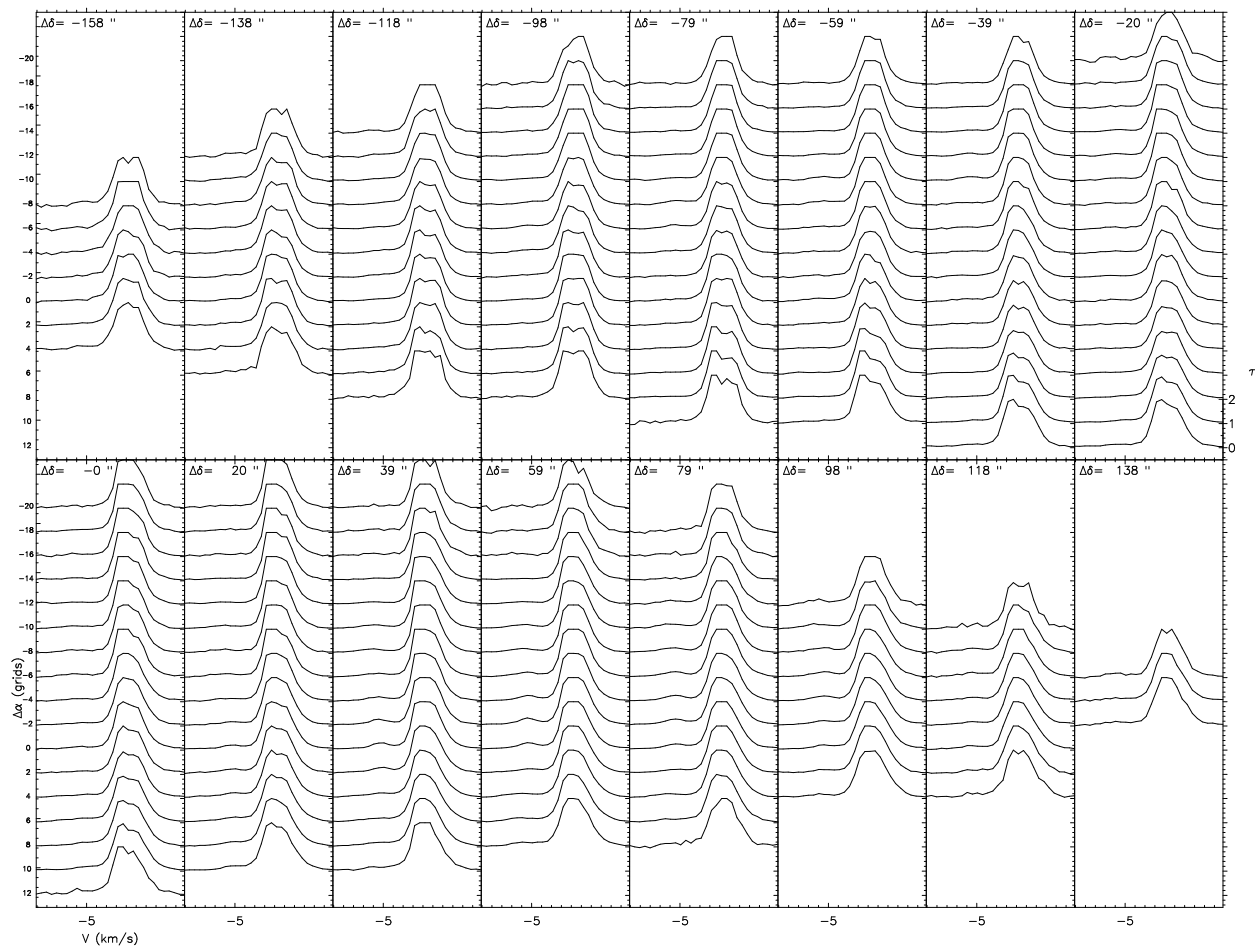

Fig. 6. As Fig. 5 but of the local gas $\left(-10<v<10 \mathrm{~km} \mathrm{~s}^{-1}\right)$ 


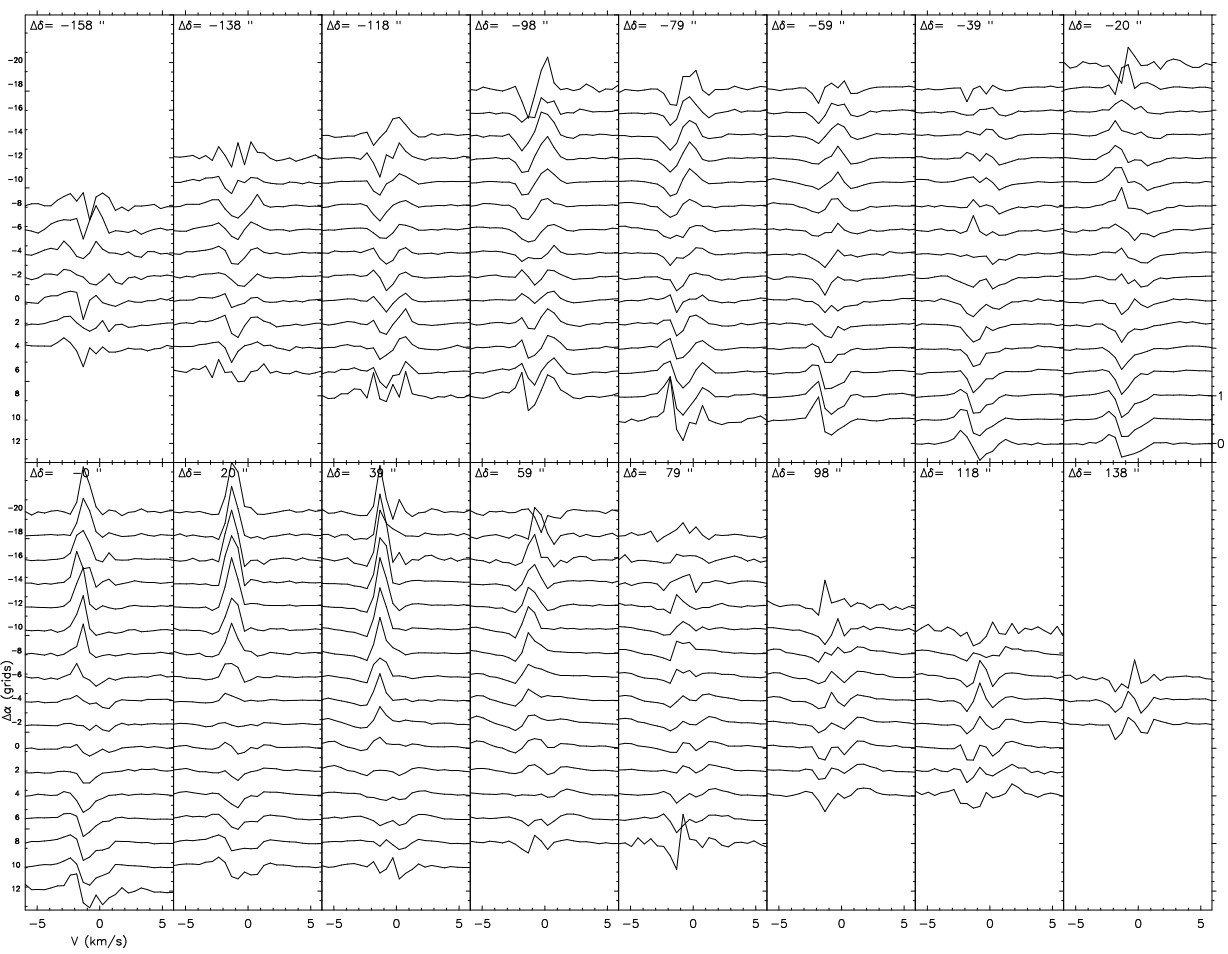

Fig. 7. HI spectra derived from $\tau$-images from which the mean optical depth has been subtracted. Because the low velocity images are quite uniform across the source, small scale features are enhanced by this presentation. Most notably is the feature at $\Delta \alpha \approx+140^{\prime \prime}$ ( -14 grids), $\Delta \delta \approx 20^{\prime \prime}$. This HI feature has an extremely small observed velocity width of $0.8 \mathrm{~km} \mathrm{~s}{ }^{-1}$ with an instrumental resolution of $0.62 \mathrm{~km} \mathrm{~s}^{-1}$. The coordinates are the same as in Fig. 5

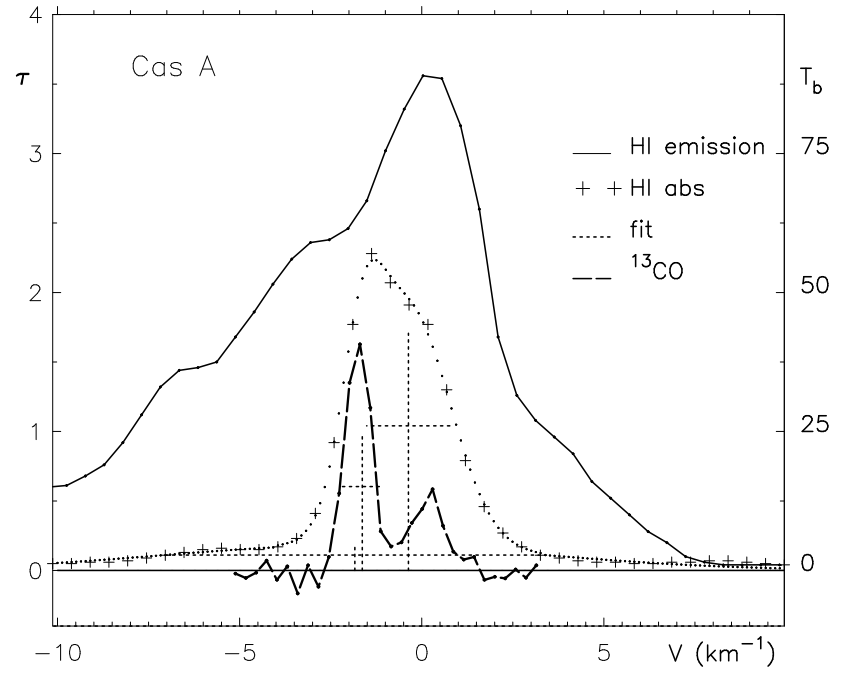

Fig. 8. Average HI spectrum in the low velocity range $\left(-10,10 \mathrm{~km} \mathrm{~s}^{-1}\right)$, symbols "+", plotted together with three fitted Gaussian (crosses) and the sum of the Gaussians, all in dotted lines. The full line spectrum is HI emission of the surrounding of Cas A, as measured by Mebold et al. (1974) and corrected for stray-radiation, Kalberla et al. (1980). The thick dashed line is the average ${ }^{13} \mathrm{CO}$ spectrum across Cas A obtained by Wilson et al. (1993); their velocity resolution was $1.3 \mathrm{~km} \mathrm{~s}$ 


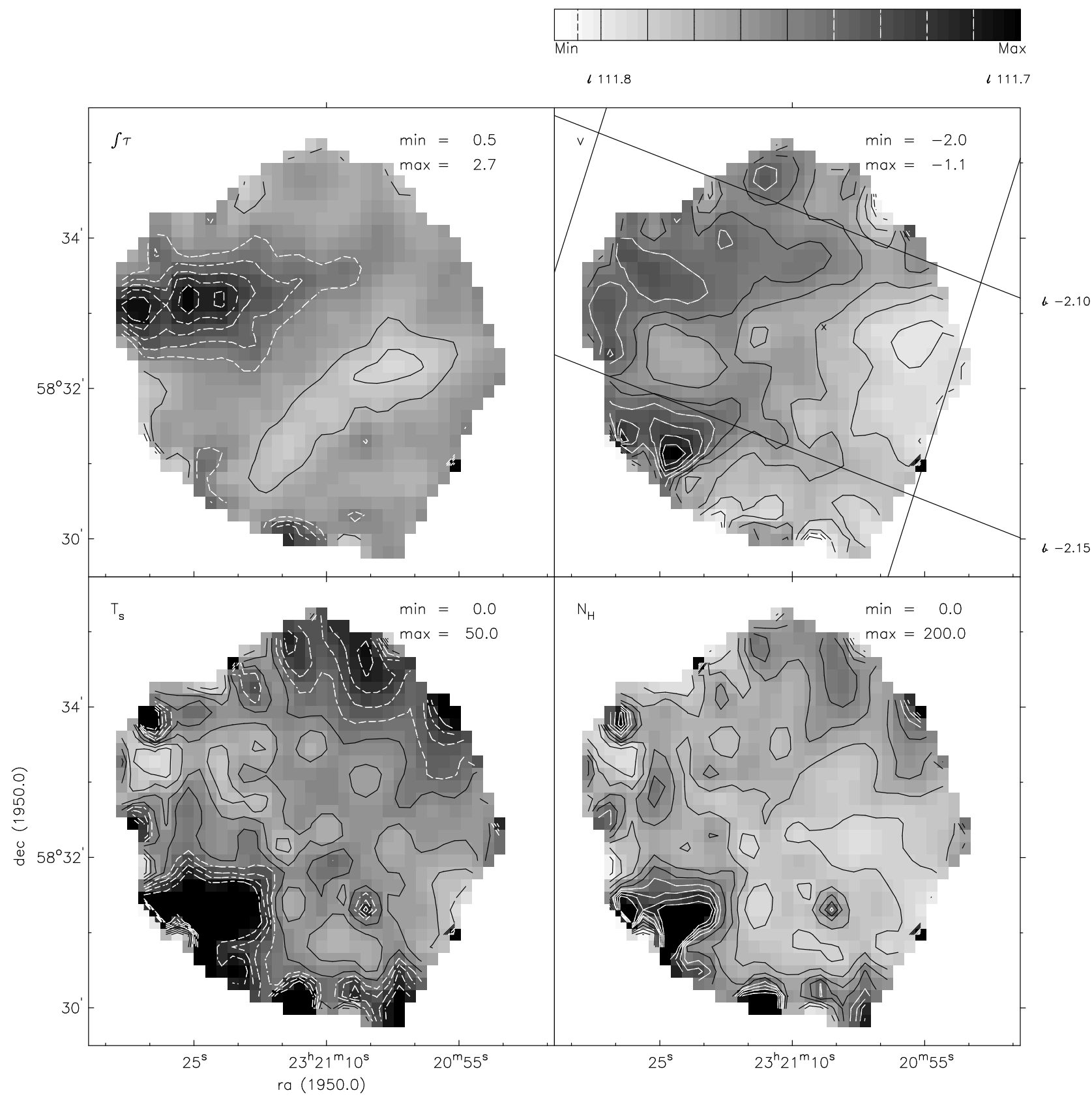

Fig. 9. Images of the parameters of the Gaussians fitted to the $-1.5 \mathrm{~km} \mathrm{~s}^{-1}$ feature. The upper two panels have units km s ${ }^{-1}$. $T_{\mathrm{s}}$ (units K) is derived from the velocity width, after correction for instrumental broadening. $N_{\mathrm{HI}}$ is in units of $10^{18}$ at $\mathrm{cm}^{-2}$ 

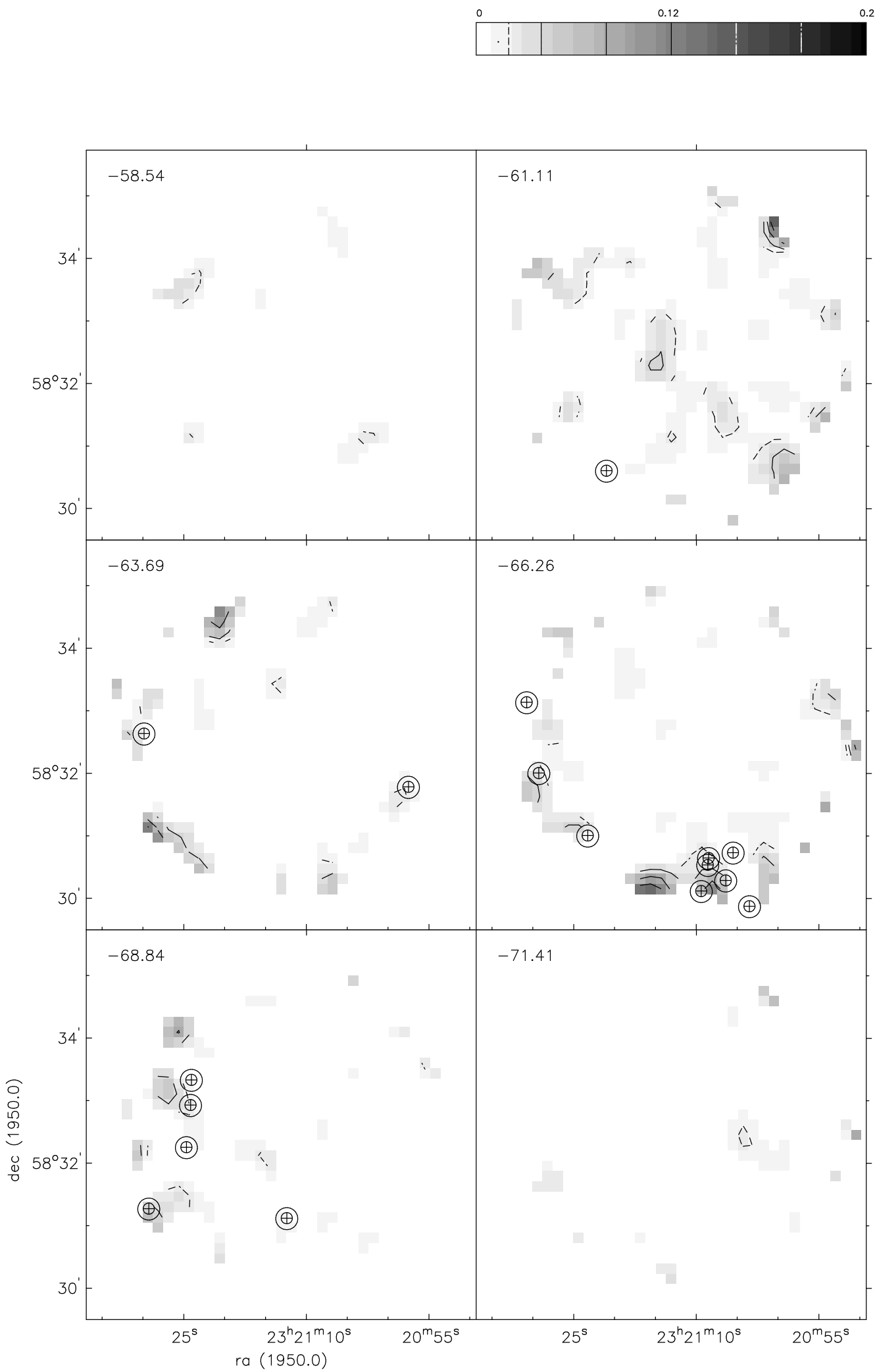

Fig. 10. Optical depth images with velocity $v$ below those of the Perseus Arm. The clumps found by Reynoso et al. (1997), are plotted as circles 